\title{
High Art: Visualising Damage on a Heritage Ceiling
}

\author{
Lindsay MacDonald \\ Photogrammetry, 3D Imaging \& \\ Conservation Collection Care \\ Metrology Research Centre \\ University College London \\ ucfs/wm@ucl.ac.uk \\ Stuart Robson \\ Photogrammetry, 3D Imaging \& \\ Metrology Research Centre \\ University College London \\ srobson@ge.ucl.ac.uk
}

\author{
lan Gibb \\ Conservation Collection Care \\ Historic Royal Palaces \\ Hampton Court Palace, London \\ ian.gibb@hrp.org.uk \\ Constantina Vlachou-Mogire \\ Conservation Collection Care \\ Historic Royal Palaces \\ Hampton Court Palace, London \\ constantina.vlachou@hrp.org.uk
}

\begin{abstract}
Photographic survey techniques were employed to monitor the condition of the paintwork on the ceiling of the Queen's Staircase at Hampton Court Palace. Illumination was provided by a flash, mounted on an 8 metre telescopic mast, raised to approximately $60 \mathrm{~cm}$ below the ceiling, with images were taken from a fixed camera position on the floor of the landing. The photometric stereo method was applied with images from ten lamp positions, to calculate surface normals and a depth map. Cross-sections at the estimated surface resolution of 7.7 pixels $/ \mathrm{mm}$, achieved a depth ( $\mathrm{Z}$ axis) resolution of approximately 100 microns.
\end{abstract}

Conservation. Historic buildings. Digital photography. Photometric stereo.

\section{INTRODUCTION}

Hampton Court Palace is a royal residence on the Thames, west of London, with a long and distinguished history. It was originally built by Cardinal Wolsey during 1514-25, with the vision of creating a Renaissance cardinal's palace, in a harmonious blend of domestic Tudor and Italian classical styles (Summerson, 1969). In 1528, as Wolsey fell from favour, he gifted the palace to Henry VIII, who embarked on further rebuilding. The following century, William III's massive expansion project intended to rival Versailles was begun, but work halted in 1694, leaving the palace in two distinct contrasting architectural styles that remain to the present day.

The Queen's Staircase is in what is termed the Baroque palace - part of a grand scheme commissioned by George II and Queen Caroline (Allen, 1984). The room is rectangular of dimensions $12 \mathrm{~m}$ by $21 \mathrm{~m}$ and has an imposing staircase rising to a landing of $12 \mathrm{~m}$ by $5.5 \mathrm{~m}$. The ceiling height is $12.5 \mathrm{~m}$ above the ground floor at the centre and $8 \mathrm{~m}$ above the principal floor landing. Daylight is admitted through three large windows and one smaller high window facing south. A large chandelier containing a cluster of tungsten lamps hangs low in the centre of the space (Fig. 1).

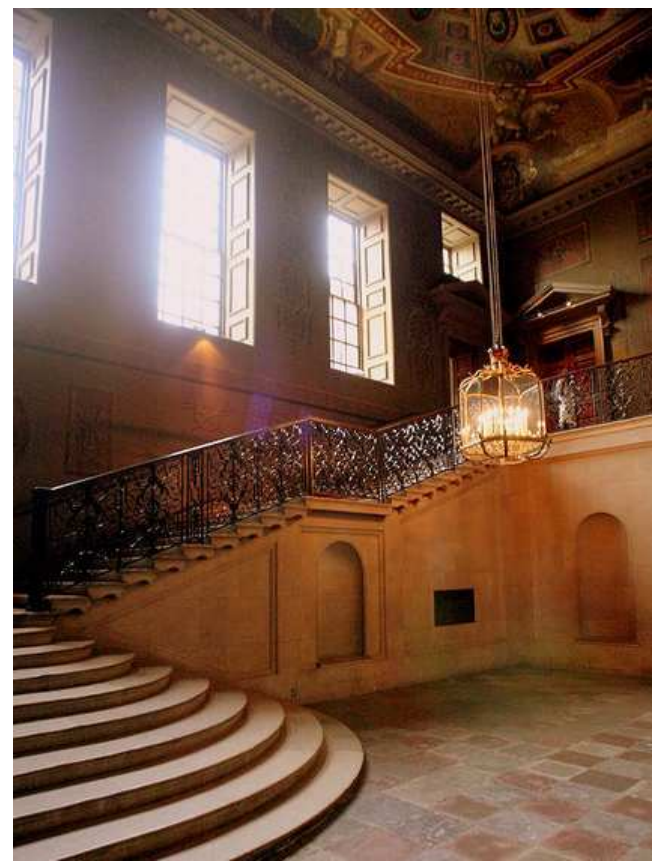

Figure 1: The Queen's Staircase at Hampton Court Palace 
The floors are paved with square ceramic tiles at ground level and close-fitting marble tiles on the landing in a hexagon/diamond pattern.

The painted ceiling, constructed in lath and plaster, is part of the decorative scheme completed by William Kent in 1734. The painting is executed in oil bound pigment and depicts a marble dome supported by a cornice and figures, featuring a central garter star. Extensive use is made of trompe l'oeil techniques with coffered panels, mouldings and elevated height to enhance the grandeur (Fig. 2). The design is centred on a Garter Star and supported by grisaille figures and elaborate ornament. According to Davies (2006): "The ceiling was painted in a consummately skilful and direct style using oil bound pigments and gilding on a lime plaster and riven oak lath support. The artist's preparation for painting comprised a considerable build-up of a red sealant layer together with a triple ground, applied in three separate layers.

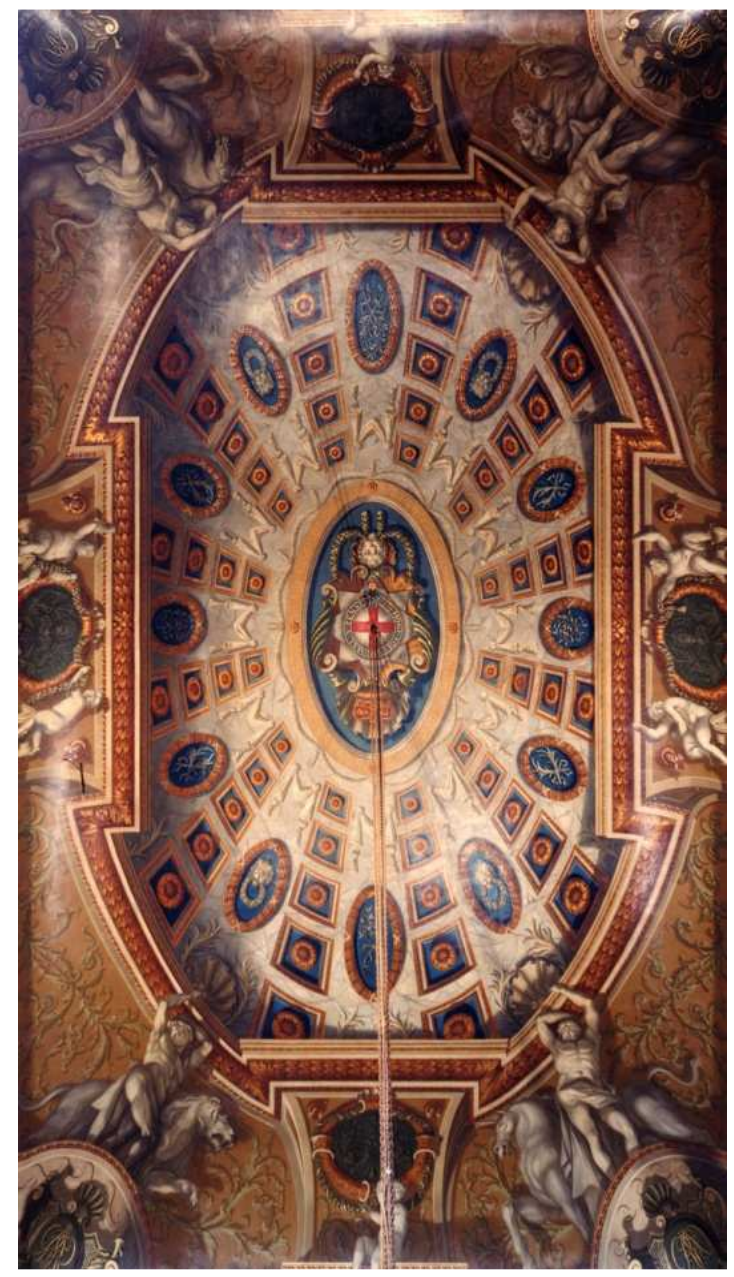

Figure 2: Rectified view of the painted ceiling

Over the preparation the design was created from a relatively simple application of paints, generally as opaque layers, with translucent paint glazes used to add depth, colour and richness to the design. Techniques for laying out of the design, such as pencil marks, incisions in wet paint and pouncing (carbon tracing) marks are present."

\section{PROBLEM OF FLAKING PAINT}

In 2001 flaking was noted on the painted ceiling. It was unlike that observed on other wall paintings at the palace, as areas of instability were spread over the entire surface of the ceiling and resulted in the detachment of large brittle flakes of diameter up to $20 \mathrm{~mm}$. Treatment with a conventional wall painting conservation material failed to address the problem. Since 2004 the conservation approach of Historic Royal Palaces (calling on the expertise of a multidisciplinary team of specialists) has been one of cautious intervention, coupled with continued research and recording to understand the causes of deterioration and to devise a suitable long-term stabilisation strategy.

Paint sampling has established the sequence of preparation layers over the previous plaster finish as follows: a red pigmented organic medium sealant, followed by three ground layers (off-white, cream, then grey). Crucially, all samples feature a loosely bound or unbound chalk layer beneath the red sealant and over the original plaster (Davies, 2006). This appears to be the interface where the delamination of the paint is taking place; even during sampling the sample flake would frequently come apart from the plaster substrate at this layer.

Treatment to improve the adhesion of the painting to the chalk layer has been investigated but has been unsuccessful due to the impermeability of the paint layers together with subsequent varnish and wax coatings. Since 2005, emergency localised stabilisation of flaking paint has taken place using Beva 371 solvent-based adhesive, shown by trials to be the most effective and convenient treatment given access and time restrictions. Treatment has dealt only with those areas in danger of imminent loss, which have been toned in to aid visual identification of new losses. In the majority of cases, paint areas treated with Beva 371 remain stable, however this remains unproven as a longterm solution. Trials have also been undertaken using low molecular weight resins to impregnate the layer responsible for delamination.

A further contributing factor seems likely to be the unstable environment in the Queen's Staircase. Environmental profiling has shown the temperature to be well buffered, but wide daily and seasonal variations in Relative Humidity $(\mathrm{RH})$ and Absolute Humidity $(\mathrm{AH})$ are caused by high air leakage to the outside through two sets of doors on the ground floor - the staircase is an important hub for visitor routes and the doors are frequently open. These large, rapid changes in $\mathrm{RH}$ are likely to cause mechanical stresses in the paint layers due to differential expansion. Tests carried out by Oxley 
Conservation and Paul Jennings in 2009 provided a series of air leakage measurements so that information could be collated on the rate of air movement. This included an inspection of the air envelope to the Queen's Staircase from the outside, as a positive pressure differential maintained inside the room allowed areas of leakage to be identified.

Attention has therefore switched to environmental control as the most promising option to improve the stability of the paint. The present one year study is investigating the effects of improving the 'atmospheric seal' of the staircase through the introduction of double doors on one external entrance, and improved draught-proofing on existing external doors and windows. Whilst the effect of environmental improvements can be easily assessed through the existing sensor network, monitoring of paint condition has always been more difficult because close examination of the ceiling requires major disruption to visitors. Previous surveys and treatment trials were done using either a cherry-picker (unstable working position and disruptive) or a fixed scaffold platform beneath the whole ceiling (expensive and very disruptive). The aim of the investigation described in this paper is therefore to develop a method of imaging the paint surface which can detect the process of flaking as it progresses and also be deployed at long range, preferably from floor level with minimal effect on public access and palace operations. By repeating the imaging procedure at regular intervals, comparison of images will provide valuable information on rates of change, such as crack growth, surface deformation and flake formation.

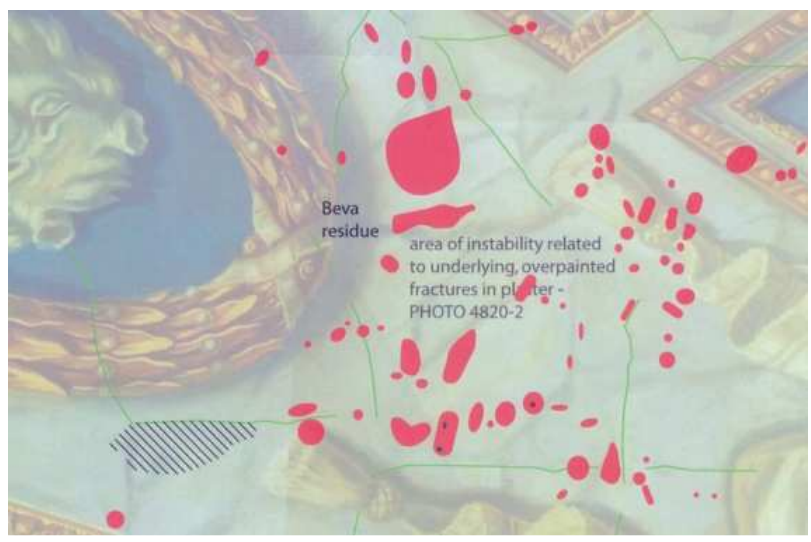

Figure 3: Map of damaged regions from survey, 2010

A detailed survey from a scaffold platform in January 2010 produced rectified photography of the whole ceiling, and mapped the regions of greatest paint loss (Fig. 3). The worst affected areas were found to be in the centre and above the landing. Possible agents of the flaking process were identified as:

- Flooding from the apartment above in 1970s;
- Thermal convection caused by the central chandelier and a ventilated heater;

- Turbulent airflow from the double doors on the ground floor and at either end of landing;

- Humidity produced by visitors and changing atmospheric conditions;

- Structural vibration (considered less likely).

The requirement of the Conservation Team is to monitor selected areas of the ceiling over a period of time to look for evidence of surface deformation, particularly bubbling or the formation of fine cracks that might precede the detachment of flakes of paint. The specified level of fine detail to be detected is $0.1 \mathrm{~mm}$ (i.e. 100 micron), which would require 20 points $/ \mathrm{mm}$ image resolution. This is beyond the capabilities of any laser scanner operating from the landing level - the typical resolution at this range would be $3-5 \mathrm{~mm}$.

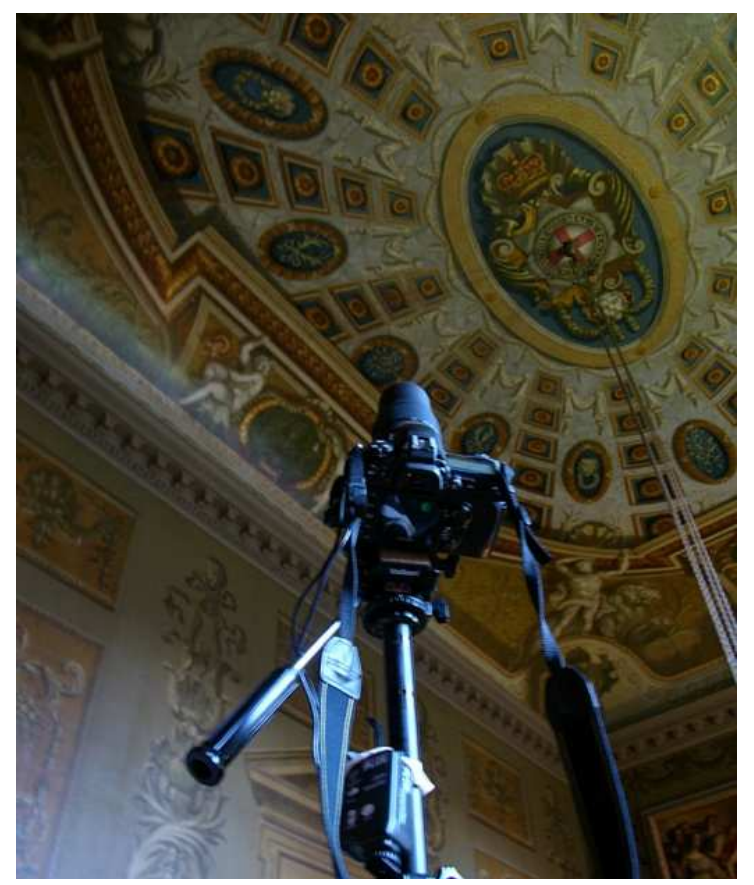

Figure 4: Nikon D200 camera with 300mm telephoto lens and remote flash trigger

\section{PHOTOGRAPHIC TRIALS}

Photographic trials were undertaken for the feasibility of capturing suitable images by digital photography from the landing. A previous study by Liang et al. (2008) with a camera mounted on a telescope was inconclusive. In this case a Nikon D200 camera with a $55-300 \mathrm{~mm}$ zoom lens was mounted on a tripod approximately $2 \mathrm{~m}$ above the landing floor level, at a distance of $6 \mathrm{~m}$ below the ceiling (Fig. 4). Images were taken directly from the camera through a USB interface into a laptop PC. The ambient illumination of the ceiling was a combination of the bluish daylight coming through 
the windows and the yellowish light from the tungsten bulbs in the chandelier. Additional illumination was provided by a hand-held flash, synchronised to the camera by a radio link. Exposure times ranged from $1 \mathrm{sec}$ to $4 \mathrm{sec}$ at ISO 400 sensitivity, changing the relative contributions to the image of the ambient and flash sources.

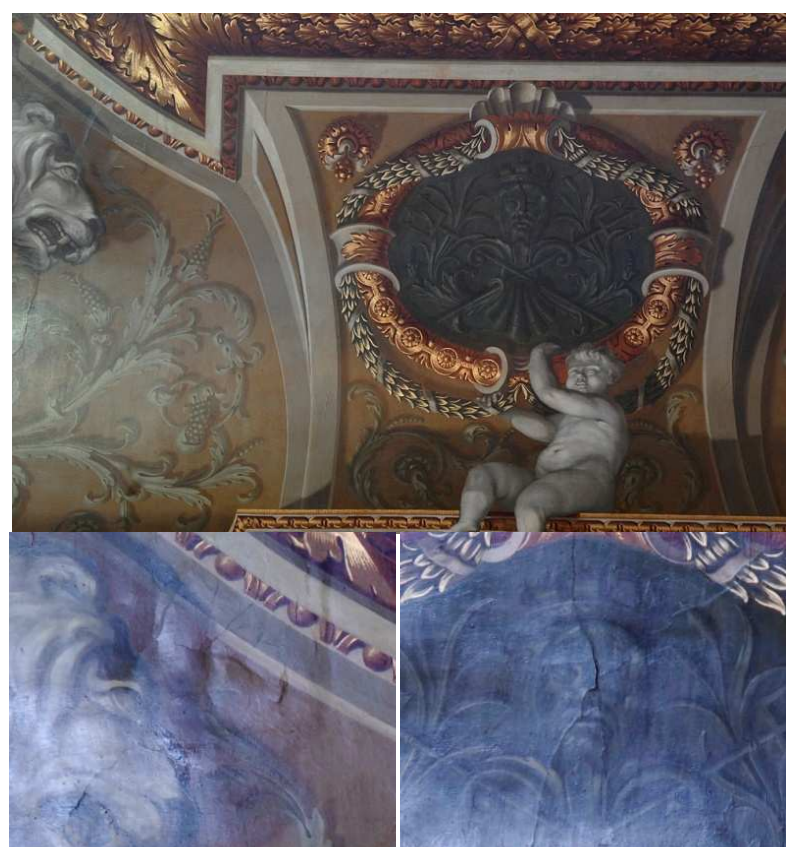

Figure 5: (top) Detail of ceiling in ambient illumination; (bottom) details of paintwork with oblique flash illumination, showing surface deformations and cracks

The ability of oblique flash lighting to show up surface detail was demonstrated in images of the ceiling adjacent to the wall, halfway along the landing (Fig. 5). These were taken with a zoom lens of $55 \mathrm{~mm}$ focal length. In ambient light (with no flash) it is difficult to observe any discontinuities in the surface. But when supplemented by side lighting from the flash, details in the images clearly show faults in the surface above the lion's head (top left of image) and in the ghostly face in the shield above the putto (centre right).

At maximum magnification of the lens (zoom set to $300 \mathrm{~mm}$ focal length) the field of view is 520'. The half-angle of 2\%0' at a distance of $6 \mathrm{~m}$ would represent a distance on ceiling of $28 \mathrm{~cm}$, so the full image of 3:2 aspect ratio would cover an area of $56 \times 38 \mathrm{~cm}$. For the image size of $3872 \times 2592$ pixels this represents a surface resolution of 6.9 pixels/mm (approx. 150 microns per pixel).

The image of one of the coffer panels (Fig. 6), taken with the $300 \mathrm{~mm}$ lens, shows two flakes, both of which are easily visible to the unaided eye from the landing level. In the image the widths of these were 118 and 92 pixels, corresponding to flake diameters of 17 and $13 \mathrm{~mm}$ respectively. Some error in colour matching (metamerism) is clearly visible in the retouched paint above the flake on the left, i.e. to the left of the gilded rosette.

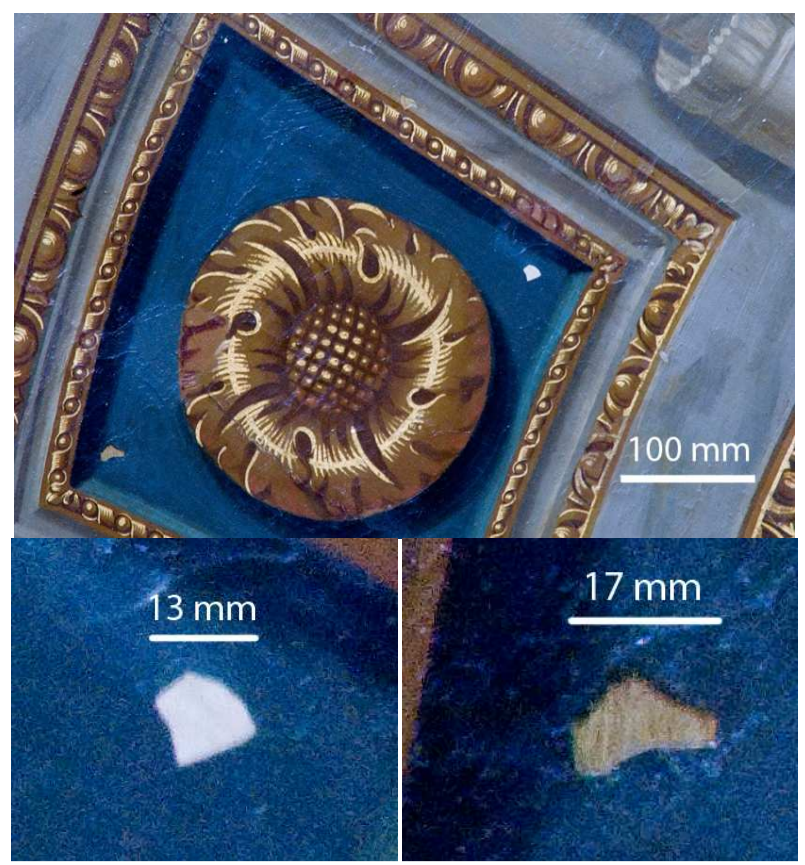

Figure 6: (top) Damage to paintwork on coffered panel; (bottom) details of areas where flakes have detached

\section{ILLUMINATION FROM TELESCOPIC MAST}

Further investigation was undertaken into the effect of raking light on the surface of the ceiling, and whether quantitative information could be derived from images captured by this means. The camera was again mounted on a tripod, at a height of 2.0 metres above the landing floor. The flash light was mounted on an $8 \mathrm{~m}$ telescopic mast, enabling it to be raised manually to approximately 1 metre below the ceiling (Fig. 7).

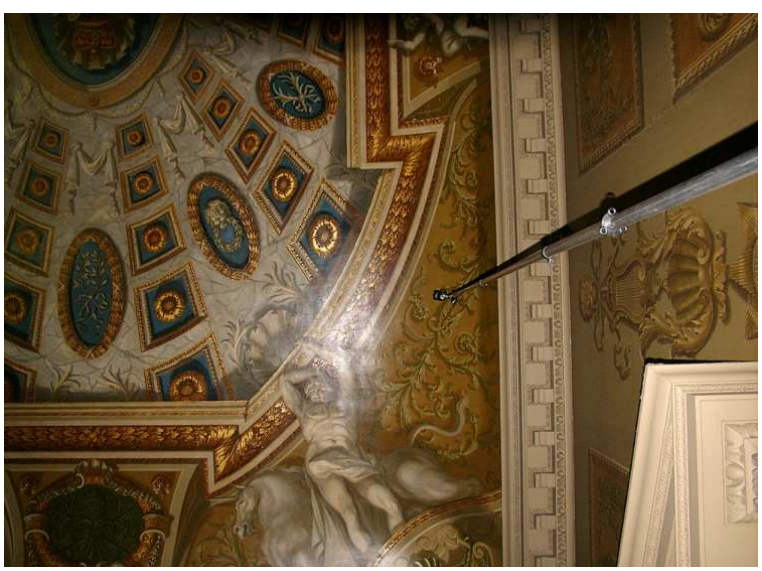

Figure 7: Mast with flash light fully raised in location 1. The target area is the oval garland on the left

The telescopic mast consists of six concentric 18gauge aluminium tubes, ranging from 2" dia (outermost) to 1" dia (innermost), each of length 1.5 metres, supported by a tripod base. When fully 
raised, the top of the mast is 7.75 metres above floor level. The flash unit and radio receiver were both mounted on the top section. The height of the mounted flash above the centre of the pole was 10

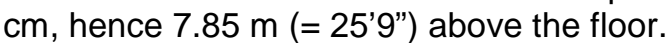

The previous rectified photographic survey of the ceiling in 2010 identified many areas subject to damage. An area in Tile 67 was chosen, depicting two branches of laurel tied by a ribbon within an oval gilded laurel garland. The target area was located on the central longitudinal axis of the ceiling above the centre of the landing rail. The camera was placed directly below, pointing vertically upwards. The flash light when fully raised was at approximately the same level as the top of the cornice. Images were taken from the fixed camera position with the $300 \mathrm{~mm}$ zoom lens, while the flash light was moved successively to eleven locations around the landing floor (Fig. 8), with five locations in each of two concentric semicircles plus one at the centre (close to the camera axis).

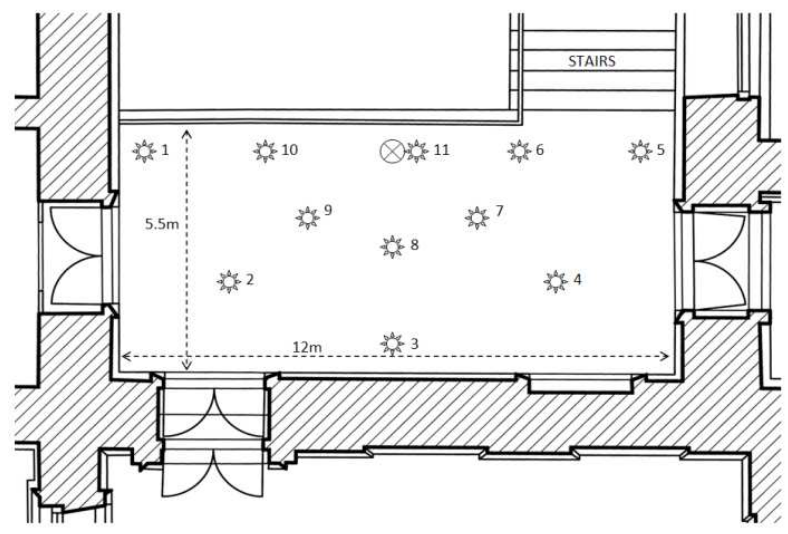

Figure 8: Floor plan of landing of Queen's Staircase, showing locations of camera and flash light

A powerful hand-held torch was used to illuminate the area of interest to assist the autofocus mechanism of the camera to operate correctly. The resulting images were sharp and revealed fine details, at a surface resolution of approx 7 pix $/ \mathrm{mm}$. Under the torchlight the images showed strong specular reflections from the glossy surface, with the visual effect of amplifying the contrast of details. Cracks, pits, brushstrokes and construction lines in the paintwork are clearly visible (Fig. 9).

In flash location 8 the light was approximately 2.25 metres from the camera axis and 1 metre below the ceiling. The rays from the lamp were therefore incident onto the surface at an angle of atan $(1 / 2.25)=24^{\circ}$. This angle provided good illumination over the whole surface while avoiding any specular components and the colour was naturalistic, rendered for flash light at 6150 Kelvin.

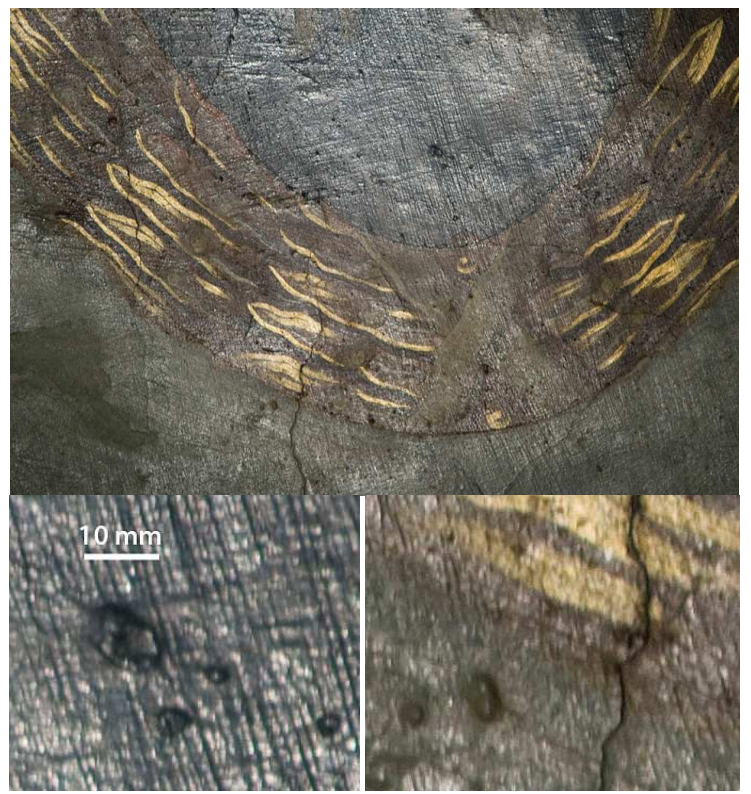

Figure 9: (top) Image of the bottom of garland, illuminated by torchlight; (bottom) details of crack at bottom and bubbling paint in centre, both of size

$350 \times 280$ pixels corresponding to $50 \times 40 \mathrm{~mm}$

In flash locations 1 and 5 the light was approximately 5.5 metres from the camera axis at a constant distance of 1 metre below the ceiling. The rays from the lamp were therefore incident onto the surface at an angle of $\operatorname{atan}(1 / 5.5)=10^{\circ}$. While not quite 'raking', this angle was low enough to show up surface features clearly, especially comparing images lit from opposite directions (Fig. 10).

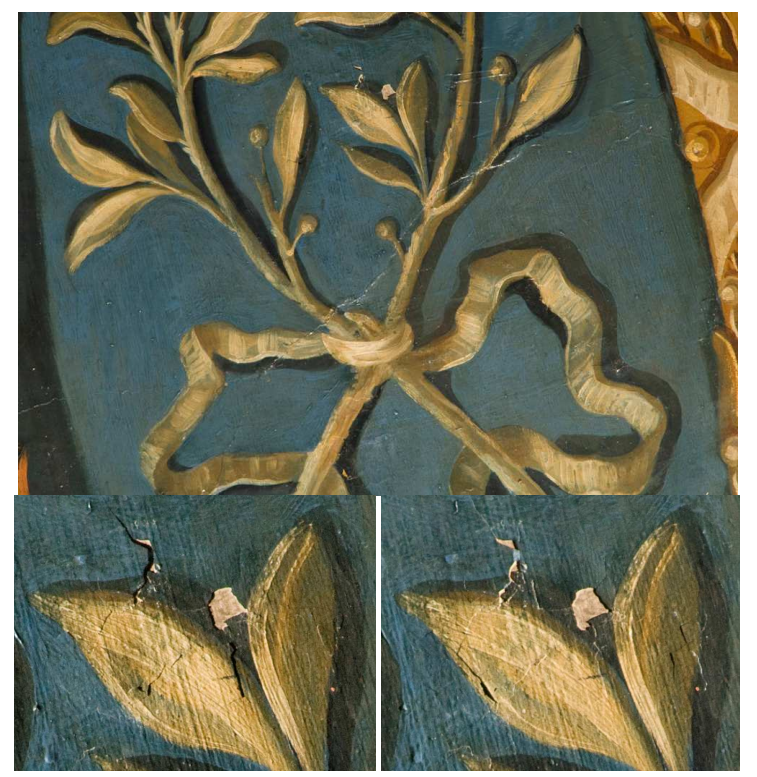

Figure 10: (top) Image taken with flash light at location 8, at 1/30 sec, f/11, ISO 250; (bottom) Details of size $700 \times 540$ pixels $=100 \times 77 \mathrm{~mm}$ taken with flash illumination from locations 1 (left) and 5 (right) 


\section{REGISTRATION OF IMAGES}

The sequence of 11 images of the oval panel on the ceiling took more than an hour to capture. Although the tripod holding the camera remained in the same position, it took an average of 7 minutes to set up each successive position of the mast holding the flash light. The high magnification of the image through the $300 \mathrm{~mm}$ lens made the process very sensitive to small perturbations of the camera and its tripod, and resulted in significant misalignment of the images (Fig. 11).

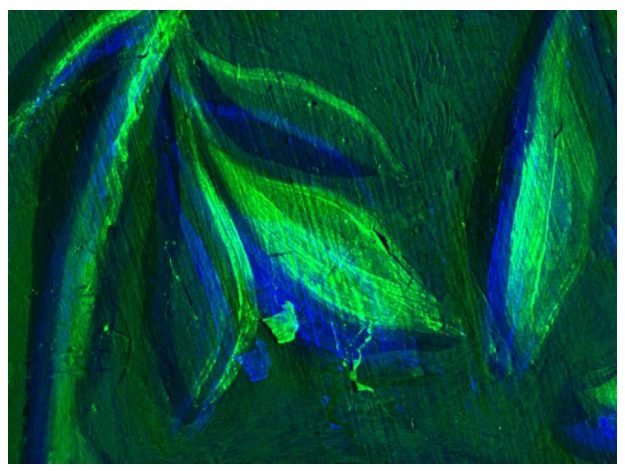

Figure 11: The effect of the misalignment of images is clear in this false-coloured composite of a detail of image 1 (green) and image 5 (blue). The offset is 45 pixels in $X$ and 73 pixels in $Y$

To use the images subsequently for making a polynomial texture map (PTM) or for determining surface normals through photometric stereo, it is essential that all images are in accurate pixel registration. In the general case of the camera moving to different locations, it would require a full projective transform to register each image to a common geometric reference frame. In this case, however, the camera movement was small enough that the effect on the image appeared as a simple two-dimensional translation. A procedure was developed to register each image in the sequence to one selected image (taken as the reference).

The images were first converted from Nikon raw (nef) format to 16-bit linear (tiff) format files, and the green channel extracted. Thus all registration calculations were performed on 16-bit linear monochrome data. The method for registration used 2D correlation, taking a small square of $101 \times 101$ pixels of the reference image, and (in effect) sliding it around an enclosing region of $201 \times 201$ pixels from the test image to find the best fit. At each successive pixel coordinate position the correlation coefficient $r$ was calculated (Fig. 12). This method has the advantage of being independent of the brightness and contrast of both images because it takes differences from the respective means and normalises the intensity values.

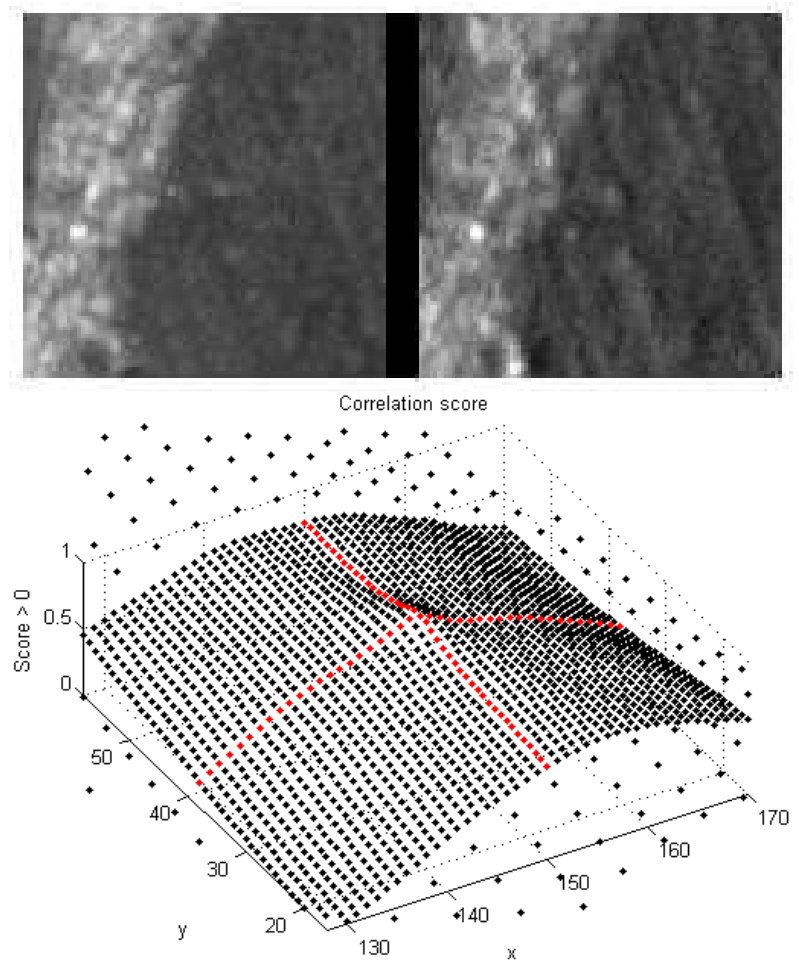

Figure 12: (top) Corresponding areas of $100 \times 100$ pixels from image 7 (left) and image 1 (right). The contrast is greater in the latter because of the lower incident angle of illumination; (bottom) Surface of correlation scores for offsets of \pm 21 pixels from the maximum. The red lines indicate the row and column of maximum correlation

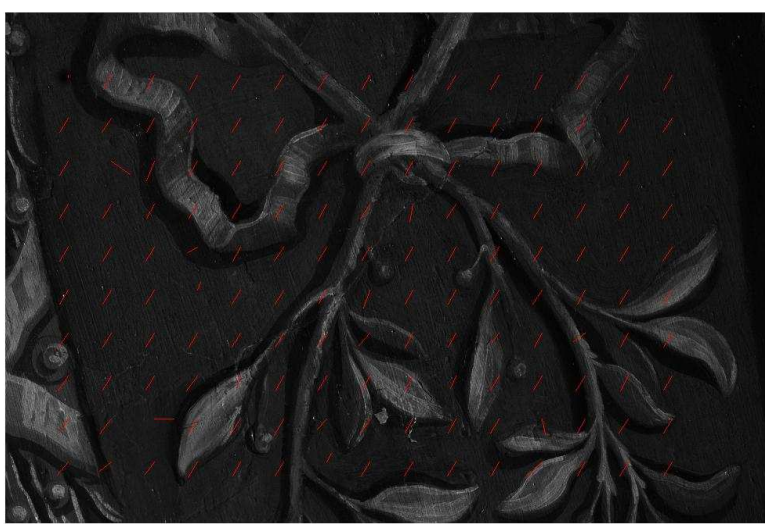

Figure 13: Monochrome of image 1 with translation vectors for image 5, calculated at 150 positions

To speed up computation the correlation procedure was performed in two stages. First a 'coarse' fit was determined by calculating at intervals of four pixels in both $X$ and $Y$ over a 201x201 pixel area, using filtered versions of both images (a $5 \times 5$ pixel mean) to reduce the influence of noise. In the second stage the fit was determined for every pixel of the unfiltered images within a local $11 \times 11$ pixel area around the maximum position found in the first stage. To make the procedure more robust, the calculation was repeated at 150 locations, on a $15 \times 10$ grid with centres separated by 220 pixels in each dimension (Fig. 13). 


\section{SURFACE FROM PHOTOMETRIC STEREO}

Photometric stereo is a technique for estimating the 3D surface from a series of images taken from a fixed camera position with illumination from different directions. It is also known as 'shape from shading', and has been applied successfully to the analysis of ancient Egyptian clay tablets, with both positive relief produced by raised moulded characters and negative relief from incised characters (MacDonald and Robson, 2010).

The green channel was extracted from each of the registered images and scaled to produce a more homogeneous image set, compensating for the different illuminance levels (because of differing lamp distances), different camera settings (f-stop and ISO and exposure time) and non-Lambertian (semi-gloss) surface.

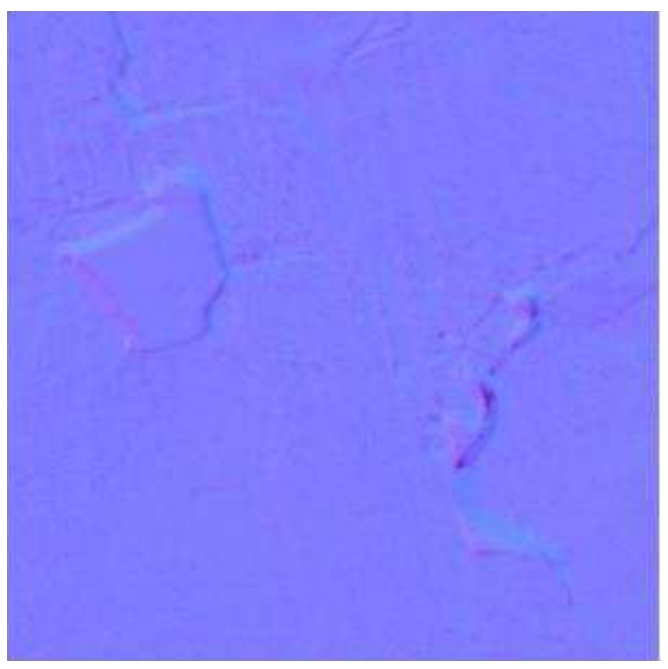

Figure 14: Representation of surface normal, where red channel represents the $X$ normal, green channel the $Y$ normal and blue channel the $Z$ normal

Images from ten lamp positions were used in the calculation. The eleventh image, with the flash adjacent to the camera axis, was not used because the high specular reflectance made it very different in character from the other ten. Although in theory there are ${ }^{10} \mathrm{C}_{3}=10^{*} 9^{*} 8 / 3^{*} 2^{*} 1=120$ possible combinations of three lamps out of ten, in practice some of these could not be used because two or three of the lamps lie in the same plane. The surface normal for $X, Y, Z$ were calculated for each of the 37 three-lamp combinations for every pixel position. The median of the distribution was taken independently for each of $X, Y, Z$ as an estimate of the true value of the normal. A false-colour image of the normal (Fig. 14) shows that the depth information is rather subtle, but the outlines of the missing flake on the left and the crack on the right are clearly visible.

Deriving a depth map from surface normals requires solving the Poisson equation by two- dimensional integration, and is not at all a trivial procedure. We followed Kovesi's method (2005), in which the surface gradients are projected onto a set of integrable basis functions. This algorithm has the advantage of a simple expression in Matlab and is highly robust to noise.

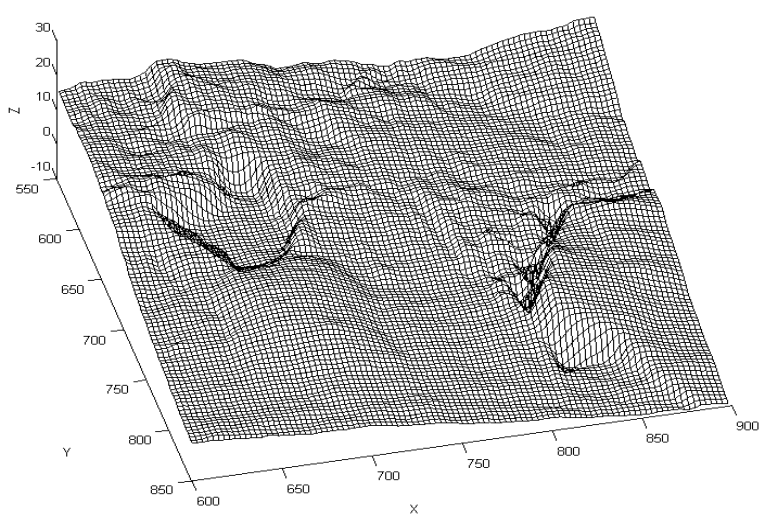

Figure 15: Reconstructed surface of $300 \times 300$ pixel area, showing flake (left) and crack (right)

The normal were corrected by finding a line of best fit through the normal $X$ data for every row and taking the median of the slope and offset values of all lines within the image region. The value predicted by the regression line across the row was then subtracted from the $X$ normal value, giving the residual for each coordinate position. A similar correction was applied to $\mathrm{Y}$ normal down each column. This procedure effectively removed the macro geometry (orientation and curvature of the ceiling relative to the camera) and retained the micro geometry (local cracks, bulges and flakes).

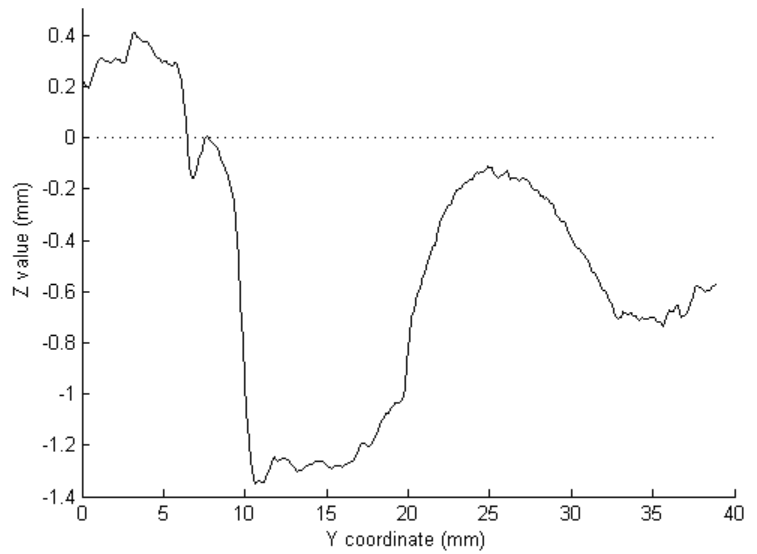

Figure 16: Vertical cross-section through surface profile after correction of normal

After surface reconstruction of the corrected normal values, the cross-sections of Fig. 16 were obtained. The axes in these figures have been converted to physical units of $\mathrm{mm}$, using the estimated surface resolution of 7.7 pixels $/ \mathrm{mm}$. Note that the depth $(Z$ axis) resolution achieved by the photometric stereo technique is finer than $0.2 \mathrm{~mm}$ (i.e. 200 microns). 
With a better lens $(400 \mathrm{~mm}$ focal length and higher MTF), combined with more careful placement of the flash light in successive positions, a depth resolution of 100 microns could readily be achieved. This is an order of magnitude better than most 3D laser scanners, and compares very favourably with the Z-axis resolution achieved by the best (and most expensive) 3D scanners, operating at a range of 6 metres (Table 1).

Table 1: Accuracy and noise of three commercial laser scanners (data from manufacturers' product sheets)

\begin{tabular}{|l|c|c|}
\hline \multicolumn{1}{|c|}{ Scanner model } & Accuracy at 6m & Noise at 6m \\
\hline $\begin{array}{l}\text { FARO Focus 3D } \\
\text { Laser Scanner }\end{array}$ & $\pm 2 \mathrm{~mm}$ & $0.5 \mathrm{~mm}$ \\
\hline $\begin{array}{l}\text { Leica HDS 7000 } \\
\text { Laser Scanner }\end{array}$ & 100 micron & 400 micron \\
\hline $\begin{array}{l}\text { Nikon Laser } \\
\text { Radar MV330 }\end{array}$ & 63 micron & 25 micron \\
\hline
\end{tabular}

\section{CONCLUSIONS}

The study proved the viability of digital photography for monitoring the condition of the paintwork on the ceiling of the Queen's Staircase. It demonstrated the value of directional lighting from a remotecontrolled flash light raised on a tall mast up to near the ceiling.

\section{What worked well:}

1) The mast proved to be a satisfactory means of holding the flash and radio receiver at the required height. It was stable enough with the tripod support to stand unattended when raised to the full height.

2) The illumination from a powerful spotlight (large torch of nominal 3 million candle power) was not only sufficient to enable the camera to autofocus accurately, but also enabled useful images to be taken directly without flash. The specular contrast enhancement of the images taken in this way showed up surface features and defects very clearly in 2D.

\section{What could have been better:}

1) The Nikon camera with $300 \mathrm{~mm}$ lens was operating at high magnification, and so any movement at all in the tripod resulted in significant movement of the image area. Because of the vibration-reduction mechanism in the lens there was no problem from motion blur, but the images from one exposure to the next were frequently out of registration. This required subsequent image processing to register them accurately (by translation and rotation) before analysis of the surface normal from different lighting directions.

2) The positioning of the mast and camera on this occasion was done rather informally by visual judgement. In future it would be better to pre-plan the coordinates and then measure and mark out the floor with tape to achieve greater geometric accuracy.

3) Great caution was expressed by the conservation staff, in case the mast should tip over with the possibility of damaging the walls or injuring the staff or public. In the event it proved to be stable and reliable, and it was always positioned so that the flash was at least $50 \mathrm{~cm}$ from the cornice.

This study also demonstrated the feasibility of using the photometric stereo technique with a set of images captured by a fixed camera and movable flash light on a mast. Local features on the ceiling could be identified, and a good estimate of their size and depth obtained, including cross-sections through the 3D surface topography. By repeating the procedure at regular time intervals (e.g. monthly) it would be possible to visualise and perform quantitative analysis of changes in the condition of the surface. The advantages of the technique are that it uses relatively cheap equipment and achieves a remarkably high depth resolution.

\section{ACKNOWLEDGEMENTS}

We credit the previous conservation studies at Hampton Court by Zoe Roberts and Sophie JulienLees, and assistance from Mona Hess at UCL.

\section{REFERENCES}

Allan, J. (1984) New Light on William Kent at Hampton Court Palace, Architectural History, Vol. 27 , pp. 50-58.

Davies, J (2006) Microscopy and archival research: interpreting results within the context of historical records and traditional practice, InFocus, Issue 3, pp. 32-45, Royal Microscopical Society, Oxford.

Kovesi, P. (2005) Shapelets Correlated with Surface Normals Produce Surfaces, Proc. Tenth IEEE Intl. Conf. on Computer Vision (ICCV'05), Vol. 2, 994-1001. Software library downloadable from: www.csse.uwa.edu.au/ pk/Research/MatlabFns/

Liang, H., Keita, K., Vajzovic, T., Julien-Lees S., Spooner, J. and Zhang, Q. (2008) PRISMS: remote high resolution in situ multispectral imaging of wall paintings, Proc. Intl. Council of Museums, Committee for Conservation (ICOM-CC) Triennial Conf., Vol. I, pp. 353-358.

MacDonald, L.W. and Robson, S. (2010) Polynomial texture mapping and 3D representation, Proc. ISPRS Commission V Symp. 'Close Range Image Measurement Techniques', Newcastle.

Summerson, J. (1969) Great Palaces (Hampton Court, pp. 12-23), Hamlyn, London. 